\title{
Zaklina Ancevska
}

MSc, University Clinic of Hematology, Skopje

Brian Richards, MSc

\section{Social work in malignant hematological diseases}

UDK:364-781

Social Work in health began in 1900 at Massachusetts General Hospital in the US. At that time the way of treating patients where previously the home care has been dominant, progressively started to change to the gradual approximation of hospital treatment.

Simultaneously with the changing of the site of treatment i.e. care, the professionals were beginning to understand that the conditions in which the patients lived and their personal problems are one of the factors for their illness and recovery.

In that same hospital center, starts a home visiting program to patients by medical students and interns, so they could have the opportunity to know the patients, the way they live and in which conditions they live in.

All this, gives a vision to Richard Cabot to create a position of a social worker in the department of health. In 1905 social work spans the hospitals in the United States at the initiative of Dr. Cabot who is considered to be a pioneer of the social work in the department health. A part of his motivation to initiate social work in the department of health and social services is the proliferation of the then specific diseases (syphilis, tuberculosis) that were requiring prolonged hospitalization, care and isolation from their families and the environment in which they live.

We conclude that psychologists are excluded from the direct observation of the patients i.e. the inability to follow the lives of the 
patients at home, their family relationships and the working conditions that are direct or indirect factors for their disease.

Therefore there is a need for the introduction of the profile of the social worker in the health department, who is going to work on the social problems associated with medical treatment. The initiators of the social workers in the health department thought they would help patients to adapt to the process of hospitalization, providing advice and information on the impact of the disease and the details of the hospital stay.

The introduction of social work in the health department opens a new aspect because we can't focus only on the pathological findings in the treatment, but on the study of the living conditions as well. Social work as a discipline was on the brink of survival, suppressed by psychologists and psychiatrists, just because of the fact that in its evolutionary development it began studying the psychological theories including psychoanalysis.

This development has made professionals focus more and more on health problems. That therapywas called communication therapy, and it was focused on the patient's emotional problems. After a few years, social work as a profession and discipline in the health department has been transformed and was seen as a fundamental component, ie how society should deal with social problems.

After a few years the position of a social worker in the health department was established in several hospitals in the United States where the focus of the work is the relationship or the link between the social problems and the disease, as well as the integrating of a special service of social services within the hospital.

The three main aspects at that time that linger throughout all these decades of social work in the health department are:

- Individual approach to patients to address their problems

- Identification and continuous interpretation of the basic concept of the social aspects of the disease and the best care to patients

- Highlighting the necessity of teamwork (interdisciplinary approach) 
Social workers need to emphasize awareness of the social work in the health department as a significant and only intervention for patients and their families. The intervention of social workers in hospitals should be based on: cost effective, therapeutically effective, family effective.

Social Work in the health department confirms its justification, because ever since 1978 it has been recognized by the National Association of Social Work as a specialized profession with specific guidelines for education and practice. Social work is a professional activity of providing assistance to individuals, groups and the community in order to improve their capacities for social action and for creating the social conditions that lead to a better quality of life.

Clinical social work is the professional application of the theories and methods of the social work in the treatment and prevention of psychosocial dysfunction, disability or handicap, emotional and mental distress. It is based on the knowledge of the theories of psychosocial development, behavior, psychopathology, interpersonal relationships, stress, and cultural diversity with special accent on the individual and its environment.

On the other side of medicine is the branch of human activity that deals with the preservation and promotion of the human health, prevention of diseases, early detection, treatment and rehabilitation of sick and injured.

Medicine covers the science of structure, the functions and the development of a human, for all situations and occurrences inside the human body and the environment that affect the health and the disease. It is defined as skill for recognition, facilitation, evaluation and treatment, prevention of the disease by improving the living conditions and promoting the physiological processes and the biological evaluation of the human. In all, it represents a professional development of those skills on individual and social level. The human in the context of the medicine is observed both in terms of the internal properties of the organism and biophysical unit which needs to be in equilibrium with the environment. The human is a product of the heritage and the environment, so the medicine studies its internal structure, but also the external factors. 
Before modern medicine are set five tasks:

- Maintaining and improving or health promotion,

- Prevention of diseases,

- Early detection of diseases,

- Timely and appropriate treatment of patients,

- Rehabilitation of the ill and wounded, and their return to the conditions for normal life and work, and as well as the support for people in need of care and assistance.

The essence of medicine is to preserve and promote health and prevent disease, and its task is to treat patients approaching them as individuals in general, not only to their disease, emphasizing the dimension of the social work and its relation to medicine.

Lately it is more and more apparent the unitary vision of the man as a complete and a complex organism, in which psychological factors can't be suit and divided from the social, nor a disease can be interpreted separately without taking into account the overall personality of the patient.

Considering that our health is one of the most important elements and attributes of our life, a precondition to carry out our daily activities, for a qualitative, full and happy life in general.

The modern understanding of health is defined by the WHO (Statute 1948), proposed by Dr. Andrija Shtampar stating, Health is a state of complete physical, mental and social wellbeing and not merely the absence of disease and inability, infirmity, disability.

The controversy about the definition of health has not been completed, although the International Conference on Primary Health Care (Alma Ata, 1978) in its first paragraph of the Declaration that has been adopted reaffirmed health as a state of complete physical, mental and social wellbeing.

In the last 10 years, more and more often this definition complements the fourth dimension, and that's the spiritual component of the overall health, which involves a sense of fulfillment and satisfaction 
with our own lives, positive values, morals and emotions towards other people and to the common good.

Through the incorporation of psychological and social criteria, the authors of the WHO not only confirmed that health and disease are multi causal, but also they suppressed the dominance of the purely medical perspective in which the absence of disease is the only criterion for evaluating the health of the individual.

Several concepts confirm that health is one of the most important components of social functioning.

Philosophy and mental hygiene concept of health means the maximum capacity of the individual for self-realization and selfachievement, taking into account the human internal strengths and possibilities, as well as the feeling of pleasure or displeasure in his behavior towards the environment.

The environmental era within the functionally oriented concept, emphasizes that human health can be complete, if while fighting for it, the man doesn't violate the health of his environment.

The social-medical approach towards health, suggests that we should not only monitor the health of the individual, but also the health of groups and the community as a result of the interaction of the individual with the social environment.

The medicine has made a real revolution in the treatment of the malignant diseases. The advance in medicine allows application of various targeted therapeutic approaches towards the treatment of malignant diseases that in general are characterized by a long-term process of treatment, frequent hospitalizations, complications that are manifested with symptomatology of various organ systems, but it should be noted that today's treatment results of these patients report the extension of the life span and improve the quality of life. This fact draws the interest not only to the effectiveness of methods and treatment but also to the biopsychosocial factors in the treatment of diseases and living with the malign nature of the disease. 
This last therapeutic modality justifies the necessity of an integrated therapeutic approach to the treatment of the patients with malignant diseases, where a separate, complementary, therapeutic modality, the work and the interventions of the social worker should be included, in terms of disrupted social relations and interactions with the community.

Malignant diseases do not occur as a result of a cause, but as a result of action among several factors: inheritance, resistance of the organism, psychological traits, etc.

According to WHO, many malignant diseases occur as a result of poor lifestyle and the effects of the external environment factors, genetic factors and infectious factors, as more studies that studied the relationship of certain risk factors include lack of exercise, obesity use of toxic substances and other.

These changes are the result of the interaction between genetic factors and three categories of external agents: physical carcinogens (ultraviolet and ionizing radiation); chemical carcinogens (asbestos, toxins); biological carcinogens (infection by certain bacteria, viruses, parasites).

During its evolutionary course, the human organism has developed a number of defense mechanisms in terms of preserving the integrity of the genetic material DNA and its preservation, defeatingthe numerous carcinogen challenges that the human body is facing daily. However the fact that malignant diseases account for a large proportion in the morbidity and mortality of our world's population indicates that the load that one has to bear in certain situations is overweight.

The main feature in the malignant transformation of cells is the occurrence of certain genetic mutations that as a consequence have caused disruption of inter and intracellular homeostatic cellular processes, which results in interruption of the process of programmed death of the cell and the acquisition of characteristics of uncontrolled proliferation, malignant neo-angiogenesis, and one of the most devastating features is the ability for metastasis of the malignant cells, which itself is the reason for the failure of therapy and small cure rates in these patients. 
Report data from the results achieved in the treatment of malignant diseases, despite the different treatment modalities have the common feature that connects all patients, and that is timely diagnosis of malignant disease. The best results of treatment and the highest rates of overall survival of the patients were achieved only in terms of early detection of malignant disease and prompt initiation of treatment.

Hematological malignancies disrupt the function of the hematopoietic system, which can lead to a multisystem symptomatology, which is often fatal for the patient.

Hematopoiesis is permanent and dynamic process of production and maturation of blood cells in the bone marrow. All blood cells originate from a common pluripotent parent hematopoiesis cell. Basic features of the parent cell are an opportunity for self-renewal, differentiation with the ultimate goal of maturation to functional cells in the peripheral blood.

Advances in molecular biology and genetics investigations are enabling more detailed understanding of the patho-genetic processes that leads to hematological malignant transformation of cells in most malignant hematological diseases. Development of modern medicine allows to accurately define the clinical picture of hematological diseases. The improvement of diagnostic and clinical investigations Para-clinics and increase the sensitivity and specificity of visual techniques and methods (x- ray examinations, computerized tomography) greatly contributes to the timely and accurate preparation of diagnostic mosaics of these diseases.

Before starting with the appropriate treatment it is necessary to assess the extensiveness of the disease, which will greatly contribute to the impairment of quality of life.

The percentage of malignant hematological diseases is $9.5 \%$ off the total number of malignant diseases. Although not included as the most common, yet stand by the complexity of its pathology, clinical picture and the undoubted need for integrated treatment approach.

Hematologists have the dominant role in the treatment of these diseases, following and implementing the modern treatment protocols and standards, but the contribution of a social worker is crucial, because the 
Journal of social policy, year 8, no.11/2 Skopje, November 2015

chronic course of illness and treatment have their own long-term impact on the social life of the patient andits reintegration in social community is especially important.

Social work promotes social changes, solving problems between human relationships, empowerment and liberation of people in the pursuit of well-being.

Using the theory of human behavior and social systems, social workers intervene in the moments of interaction of people with their environment.

Living in a time of rapid and complex social and political change, in a time of economic crisis and valued, progression of unemployment, poverty, disease and the occurrence of another, at much of the population leads to disruption of individuals, families and groups as a result of numerous social pathological changes.

Increased social problems and social risks in society have increased the need for the establishment of professional and technical assistance for their solution i.e. most directly engaging social work.

The main objective of the experts in the field of social work is based on helping individuals and families, or to intervene in the interaction of the individual and the environment, particularly in the situation in it.

Disturbed relations between the individuals and the environment are having a negative impact on the health of the individuals, especially on mental health or psychosocial functioning. This gives rise to the indication for implementation of social work in the area of health in primary, secondary and tertiary prevention of health. The social worker in the field of health care is focused on helping patients with psychological and social problems caused by diseases and associated with frequent hospital treatments. Emphasis is placed on the functional disturbances at the healing process, and not directly on the disease.

In providing psychosocial support social worker pays attention to the interaction between psychological and social components that affect human functioning. It is aimed to protect and restore or enhance the 
relationship between a patient and his social environment that helps his reintegration.

The attitude towards the patient is based on respect for his personality and to gain a clear insight into his own condition, detection of social problems and to act for their dismissal.

With regard to the implementation of this activity in the field of health, social worker perform tasks through mediating, referral and counselling talks, providing technical assistance in the realization of rights in the area of social and health care, create and mediate for hospitalization in the relevant institution.

The fact is that patients with malignant haematological diseases encountered numerous as physical and psychosocial problems. But the progress of medicine in the field of malignant haematological diseases in the last decade has seen significant growth.

Information on the disease leads to a shock for both the patient and members of his family, because there is always associated or connected with the near death. Psychosocial problems at patients with malignant haematological diseases can be different depending on interaction complex medical, psychological and social parameters.

On one side the nature, type, intensity and duration of stressed events caused by malignant diseases, and on the other side are the ways in which the patient and family experience them.

Almost all patients go through a similar phase when faced with illness and complications that may occur during the course of the treatment. Facing the diagnosis and the needs for treatment, patients quit their work, education, ie the community they belonged in, for a hospital way that changes their previous lifestyle. The fear of death, the presence of weakness, pain, loss of dignity, self-respect and the preoccupation with the new situation and uncertainty, make social contacts difficult on a wider scale that leads to social isolation of patients.

Long and frequent hospitalizations of the patients with malignant diseases cause changes associated with costs that are necessary for their treatment, which increases the material difficulty and brings new 
uncertainty in the domain of the family. More research that were made in this area and some scientific achievements reported that patients with greater social support and patients who use strategy to overcome stress such as focusing on positive events and distancing from the negative aspects of the disease, record better reintegration into the community and better acceptance and coping with the disease.

Those kinds of experiences affect the establishment of rehabilitation programs for patients, where the size and facilities which are realized in the last decades is increasing rapidly. Rehabilitation programs that are designed for this population are focused on social support, including interdisciplinary team, such as: social workers, clinical psychologists, health professionals, including a variety of medical, psychotherapeutic, behavioral and other interventions. Interventions are undertaken in order to reduce the negative side effects of chemotherapy (nausea, vomiting) emotional difficulties (depression, anxiety) and pain as an important factor for the functionality of the patients. The goal of the implementation of these interventions is to facilitate and render better the everyday of the patients, and thus improve their quality of life.

Behavioral interventions and cognitive behavioral approaches are used to establish an individual control over the patients associated with specific problems that follow the illness and the treatment, particularly in the reduction of pain, anxiety and cognitive restructuring.

This intervention involves patient education related with the course of the illness, the method of treatment, the diet and the overcoming the problems associated with long and frequent hospitalizations.

Psychosocial interventions in rehabilitation programs that are designed to meet the psychosocial needs of the patients with malignant hematological disorders and their family members include approaches to overcome them.

Individual therapeutic treatment applies to patients after the diagnosis is final and at the beginning of the treatment, where psychosocial problems are at their peak. The presence of anxiety, depression, suicidal ideas, fear of death, fear of the changing physical appearance, as a result of the application of the cytostatic therapy, which 
results in impaired social relationships to progression of social isolation. The problems associated with the treatment of malignant hematological diseases and long-term hospitalizations are manifested in loss of concentration, weakness, loss of appetite, immobility or progression of the disease, which leads to intervention in the crisis, focus on specific problems and their resolution, uncertainty and losses that follow the life of the affected.

More than two decades, therapists, social workers, clinical psychologists and health workers combine drug treatment with psychosocial interventions, not only in order to meet the psychosocial needs, but also to increase the level of motivation for the further course of treatment. The main objective of this application is to improve the prognosis and quality of life in patients with hematologic illnesses.

The application of social work in malignant hematological diseases and investigation of the factors that influence the expression of its application in the daily functioning of the individual within the changed framework of action, as the example of diagnosed and treated cancer, represents an additional, mandatory target in full assessment of the achieved treatment response and functionality of the individual in the social environment. The need for a clear definition of the interactions patient-disease and therapy-quality of life and clear definition the segments where we can effectively act in order to achieve positive progression towards improving the qualitative features of this category is a clear and primary goal that must be implemented in the current approaches of monitoring the patients with malignant diseases.

Due to the chronic course of malignant hematological disease, social work is present in more segments of their treatment, following the aspects of hospital treatment.

Regarding the distribution of patients by age, based on the practical operation the impression is that the advanced age at diagnosis of patients of the disease is a significant negative factor that affect on the treatment. Often in the initial evaluation of health status and the determination of the complications of underlying disease, the degree of disability or difficulty in performing basic activities confirm the fact that these features are with 
greater frequency at the elderly, social worker act of involving family members, with timely obtaining information patient through direct and mediating role.

Malignant hematological diseases require long-term treatment and complications of the disease in some patients expressed to the extent that cause disability, or a fully functioning and independent individual is transformed into a person with impaired performance status of high degree. In most of the patients as a result of the underlying disease contributes reducing their performance, and in severe cases a complete work disability. Patients with more severe stage of the disease, which results in frequent and lengthy hospitalizations, and thus a need for a multidisciplinary approach to the treatment system including social work as important in this segment of tretman.

The primary goal of therapy in hematological malignancies is achieving a complete remission of the disease, ie the absence of clinical signs of disease. It is not always possible, so in many cases and partial treatment response is accepted as satisfactory if it provides good control of the disease.

Considering that the symptomatology of the disease affects an overall figure, ie the physical, emotional and social aspects are almost dysfunctional, where inevitably proves necessary social work as dominant in all these aspects.

When we talk about physical activity and when this functionality we associate with impaired health, today almost does not exist an institution that provides health care and their recommendations for the preservation and promotion of health does not emphasize the need for regular physical activity. Physical activity is important in the rehabilitation of many diseases, but it is clear that it mostly depends on the underlying disease pathology.

Often when conducting initial interviews with patients, the announcement of the diagnosis and treatment plan for patients develop emotional disorders, mostly anxiety and depression, affecting the physical and psychological health, thedisease patients experience as mental stress, including in the fight for the main goal-good health. In practical work 
emotional disorder patients express through inexplicable sadness, denial of the new situation, fear and uncertainty about their future, and anxiety which is followed by an intense and uncomfortable feeling of tension and anxiety.

The consequences, complications, presence of pain and other symptoms of the main disease, co morbidity lead to social isolation of patients, because they have limitation in movement, disruption of activities, or reduced physical and working ability. In this section especially prominent is the contribution of the social worker, carrying a range of social skills and methods of operation. Through counselling approaches as part of the treatment team, the social worker insists despite subjective discomfort of patients to fully communicate with, including family members. The realization of an open and positive communication with patients and their families is important to realize the healing process, because cooperation with the patient is important in making crucial decisions about his health. Acceptance of the disease, facing newly situation facilitates access both medical and social perspective.

Hematological malignancies treated with transplantation of hematopoietic stem cells and represents a major advance in the treatment in accordance with predetermined criteria and indications for its implementation. Transplantation may be allogeneic HLAfrom identical compatible donor and autologous transplants from the own stem cells. Patients undergoing chemotherapy treatment process, are achieving a complete or very good response to therapy, depending on the disease and assessment of the doctor patient prepare for such an approach to treatment.

This approach in the treatment of these patients has included social work, primarily in the inclusion of the family in deciding on this kind of treatment. Allogeneic transplantation requires direct involvement of parents, biological siblings in the classification that analysis must be realized in certain hematologic diseases, after prior assessment by a doctor. The need for this type of analysis is important due to a potential identical bone marrow donor, planning the further treatment. But often we are witness of the dysfunctional family relations that makes it more difficult to deliver. Therefore, the role of the social worker is to mediate in 
communication with both patients and families emphasizing the importance of transplants hematopoietic stem cells in the context of treatment. Timely information and maintaining a healthy and open communication contribute in cooperation, the therapeutic treatment can be carried out appropriately and promptly.

Malignant diseases have social and medical significance and because of their multi-causality and mass, where the weight of the disease leads to health, social and economic consequences for both the patient and the family and social environment. In search of the literature expressing and emphasizing the importance of social work in health American Health Association defines discharge or DP as interdisciplinary process led by the following essential elements:

o Anamnesis patient data and determining the needs for post-hospital care

o Indication for the patient about care

o Educating patients with families

o Planning, development and coordination of social resources needed to provide continuous care after discharge

o Planning of financial support to enable conditions for further care.

It is for this reason one of the main objectives of the health systems of many countries should be programs for prevention of these diseases, or programs for screening at-risk population groups defined according to modern research on epidemiology, incidence and prevalence of malignant diseases.

One of the main objectives each country's increasing economic standard, and thus the level of quality of living can be improved with the introduction of public health programs that have a preventive character. 


\section{References}

Abramson, M. (1981).Ethical dilemmas for social workers in discharge planning. Social Work in Health Care, 6 (4), 33-42.

Advances in Nursing Science (2006; 29 [4], 340-50).

Стојчевски Т. (2000) Хематологија.

Flaherty, S.(2004). The role of the social worker in a physical medicine and rehabilitation setting. East Meadow NY. Nassau City Medical Center.

G. Vuletic, A. Mujkic, Što cini osobnu kvalitetu života: Studija na uzorku Hrvatske gradske populacije, Škola narodnog zdravlja A. Štampar" Medicinski fakultet Sveucilišta u Zagrebu, 2009.

Hepworth, D,. Rooney, R., \& Larsen, J. (2002). Direct social work practice $\left(6^{\text {th }}\right.$ ed). Pacific Grove, Ca Brooks/Cole.

Iglehart, A. (1990). Discharge planning Professional perspective versus organizational effects. Health and Social Work, 15(4), 301-309.

http://www.who.int/mental_health/media/68.pdf

Kadushin, G., \& Egan, M., (2001).Ethical Dilemmas in Health Care: A Social Work Perspective.

Rosen LS, et al. Cancer. 2003;98:1735-1744.

Salajka F., Quality of life of oncological patients. Czech SlovClinOncol, 2001; 1: 27-9. 
